\title{
Nonpotentiality and energy transport in NOAA AR 10720
}

\author{
Meng Zhao and Jingxiu Wang
}

National Astronomical Observatories, Chinese Academy of Science. 20A, Datun Rd. Chaoyang Dist, Beijing 100012, China

email: zhaomeng@ourstar.bao.ac.cn,wjx@ourstar.bao.ac.cn

\begin{abstract}
Based on photospheric vector magnetograms obtained at Huairou Observing Station and BBSO, we studied the evolution of magnetic nonpotentiality and energy transport in NOAA AR 10720. Daily changes of vector magnetic field was analyzed. Shear angle, helicity and free energy density which were deduced from the data were examined.

A New EFR on January 13 brought in magnetic nonpotentiality strong shear angle, free energy and complexity(multiple neutral line and opposite sign helicity) which touched off AR activity, support the idea that upper atmospheres critical state may be made by continues changes on the photosphere.
\end{abstract}

Keywords. nonpotentiality, vector magnetic field, shear angle, helicity, free energy

\section{Introduction}

AR 10720 was the most impressive group on the visible disk on Jan 2005. The region rotated onto the disk as a simple beta group on Jan. 10, grew rapidly and fully developed on Jan. 15, ended as a magnetically complex sunspot region on Jan. 22. From Jan. 14 to 21 it produced 5 X-class and 16 M-class flares, of which four were significant: X2.6 flare at 15/22:49 UT, X3.8 flare at 17/09:52 UT, X1.3 flare at 10/08:22 UT and X7.1 flare at 20/06:46 UT.

From the MDI magnetogram movie we could identify 3 main emerging flux regions (EFRs) emerged approximately from 12/02 UT, 13/17 UT and 16/05 UT. Of the 3 EFRs, the one on Jan 13 was the most prominent and plays an important role in the AR evolution. The orientation of this EFR was almost perpendicular to primal main magnetic axis and it grew very fast. After emerging it made the neutral line become longer and it created multiple neutral line. Afterward, multiple neutral line disappeared, two area of obvious cancellation took place in the west from Jan. 15 to 17 and in the east from Jan. 16 to 20. Big events all happened after EFR on Jan 13.

\section{Daily evolution of vector magnetic field}

We merge data from both Huairou observation and BBSO to a continuous tone sequence of vector magnetograms. We use first potential and then linear force-free approximation to remove the $180^{\circ}$ ambiguity of field azimuth. In Figure 1 vector field on Jan. 13 had simple bipolar configuration. On Jan. 14 neutral line became multiple. Transverse filed was complex and sheared. Flux on northwest was impacted by the EFR on Jan. 13 and showed strong magnetic shear. On Jan. 15, when the first big flare took place, the neutral line grew in length but reduced the complexity in the west, by magnetic cancellation. In the east, strong shear and complicity lasted until Jan. 20. On Jan. 16 multiple 


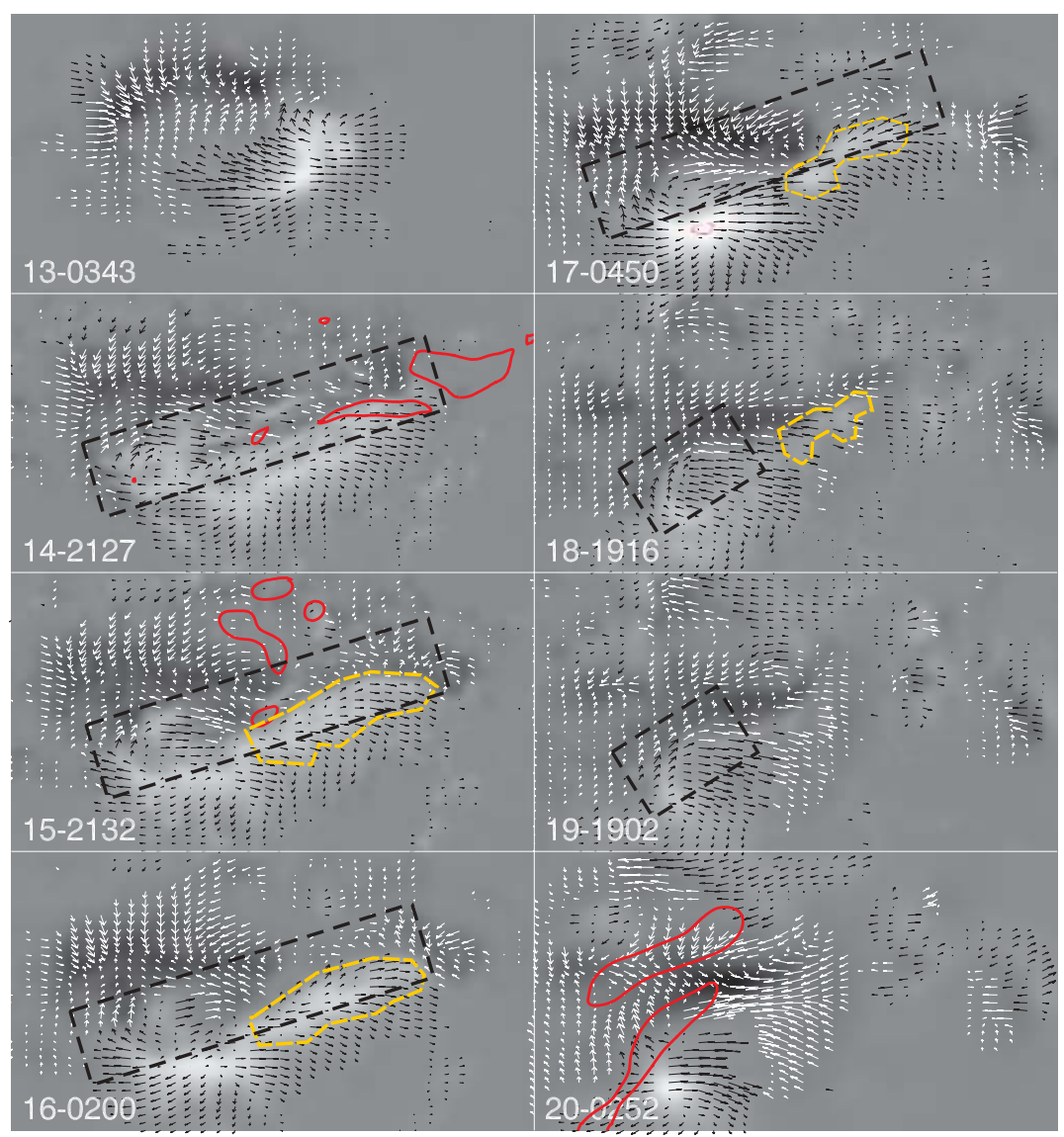

Figure 1. Time sequence of vector magnetograms with removed ambiguity of azimuth. Background is line-of-sight components. Dashed rectangles indicate the neutral line area. Flare ribbons are presented by solid contour. Area inside the lighter dashed line are used to calculate flux disappearance.

neutral line continued to disappear. On Jan. 17 many magnetic flux disappeared in the west and multiple neutral line left only a little piece. On Jan. 18 neutral line finally fragmented. From Jan 15 to 18 , more than $5.6 \times 10^{21} M x$ positive magnetic flux disappeared. On Jan. 20, because of large project effect, observation is hard to interpret. We guess that events on Jan. 15 and Jan. 17 were correlated to the strong shear, disappearance of multiple neutral line and flux cancellation on the west, events on Jan. 19 and 20 might correlate to complex vector field structure and strong shear angle on the east.

\section{Nonpotentiality distribution and evolution}

Average shear angle along neutral line always indicates main nonpotentiality of AR (Hagyard et al. 1984; Leka et al. 2003a, b) as in Figure 2. We could see two periods of obvious strong shear, Jan. 14 to 16 and Jan. 19 to 20. Jan. 15 flare took place in the declined phase of shear changes. In Figure 2 the shear change on west part of neutral line wasn't the dominant part, that's because both shear angle and the length of neutral line with strong magnetic shear indicate the magnetic nonpotentiality. The west part of neutral line was with less shear but longer. Average shear angle is about $54^{\circ}$. The neutral 


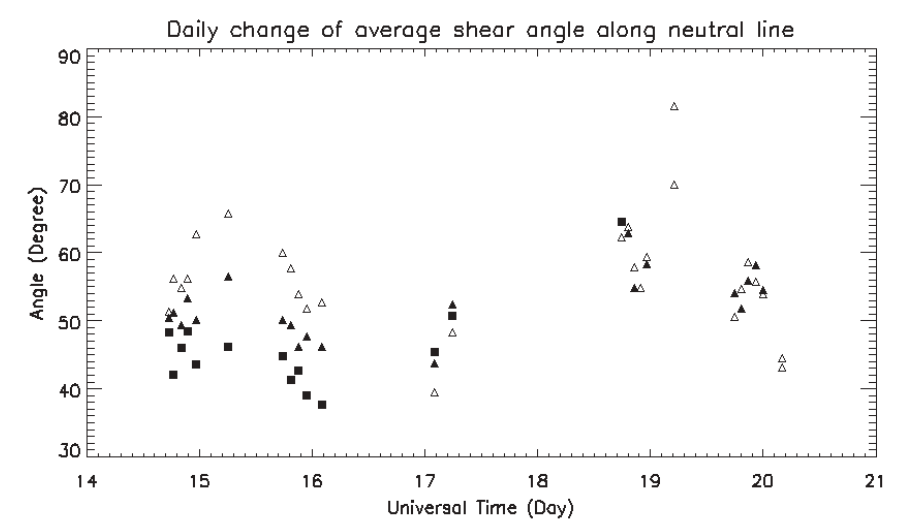

Figure 2. Changes of average shear angle along neutral line. Arrows indicate the time of big flares. Solid triangles indicate average shear angle along the whole neutral line while hollow triangles(squares) indicate average angle along east(west) part of neutral line.

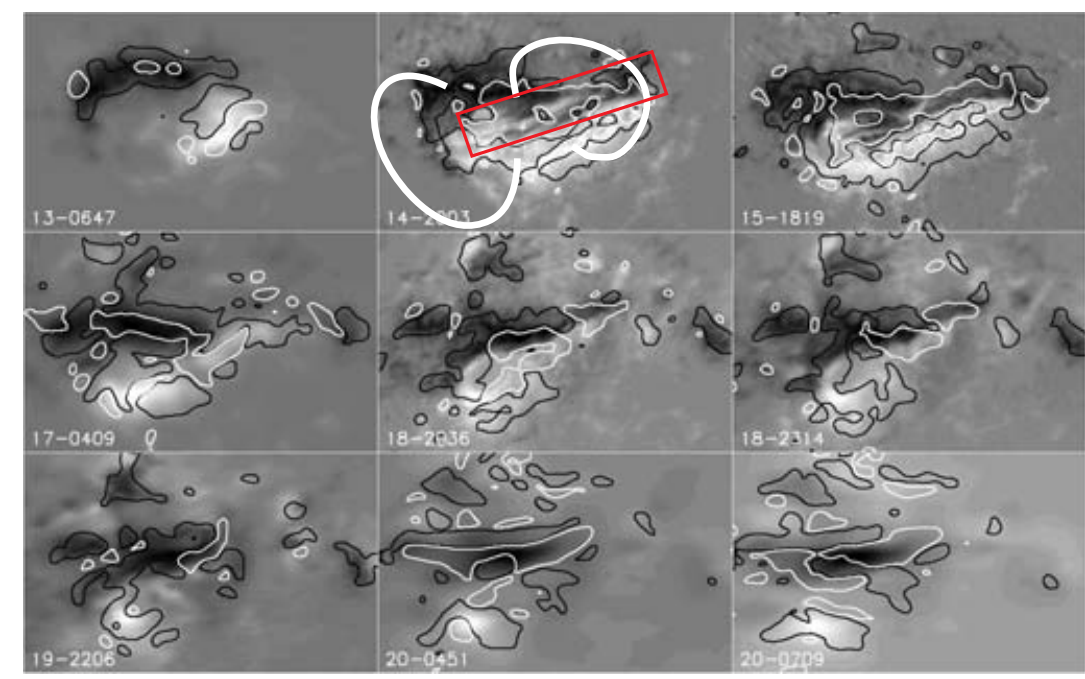

Figure 3. Daily changes of helicity. Background is line-of-sight magnetic field. Black(white) contour shows the negative(positive) helicity system.

line reached its longest on 17:00 UT Jan. 15 with a length about $110000 \mathrm{~km}$, at that time average of shear angle is about $52^{\circ}$.

Two flux area which have same sign of helicity may belong to the same system, while two area which have opposite sigh of helicity have the possibility of flux cancellation (Wang et al. 2004). Figure 3 shows that AR 10720 contained two main flux systems. The EFR emerged from Jan. 13 went upon the stage as the third system with negative helicity, which made the positive system broken up from Jan. 15 to later days. The positive helicity system also cancelled with the east part of negative helicity system which might be associated with the positive magnetic flux of the EFR. From Jan. 19 to 20 there was a interesting rebuild of positive helicity system. Current helicity was used here because that current helicity could well indicate the interaction of opposite twisted systems (Wang et al. 2004).

Free energy(Wang et al. 1996; Deng et al. 2001) is energy stored in the nonpotential magnetic field. In Figure 4 maximum free energy density concentrated on neutral line 


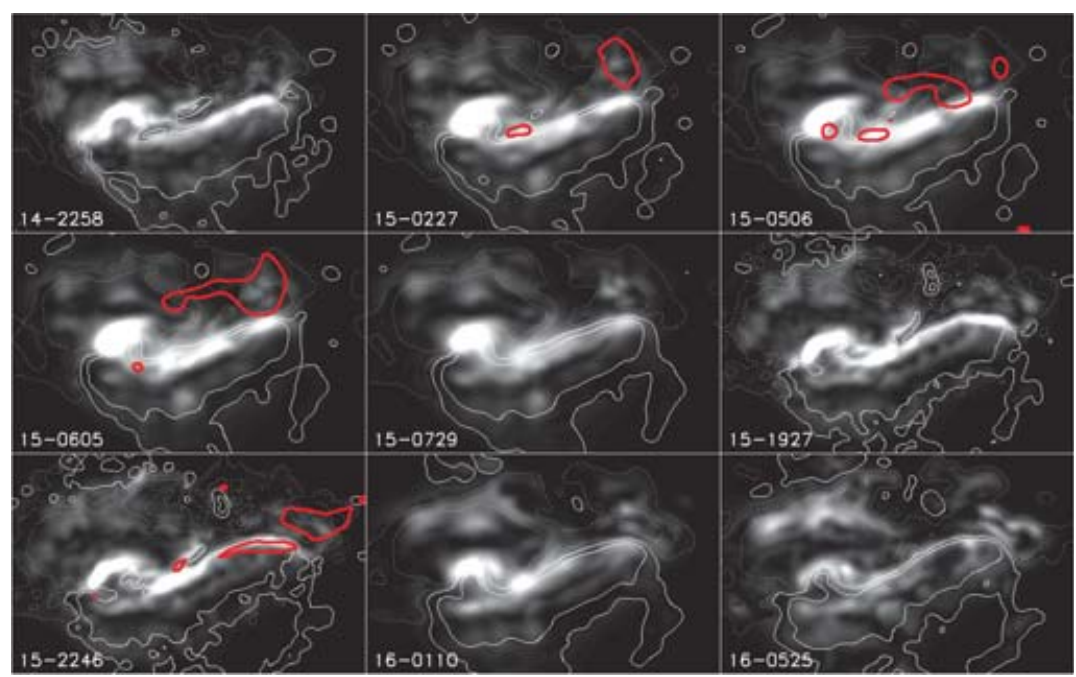

Figure 4. Free energy density changes on Jan 15. The contours represent the gross structure of line-of-sight magnetic fields. Darker contours are flare ribbons just like in Figure 1.

area and have temporal correlativity with flare. But flare ribbons do not coincide with free energy density site. Free energy density reduced after flare.

We found that on Jan. 20 shear angle and gradient of line-of-sight magnetic field decline from a quite large value. Although because of the large project effect observation is hard to interpret, we could see notable flux cancellation in the east before the event (Zhang et al. 2001).

\section{Summary}

New EFR brings in nonpotentiality including strong shear angle, and free energy, brings in magnetic complexity including multiple neutral line and complicated helicity pattern, touchs off AR activity. Cancellation from Jan. 15 to 17 in the middle east correlated to Jan. 15 and 17 events. Cancellation from Jan. 17 to 20 in the west correlated to Jan. 19 and 20 events. Upper atmospheres critical state may be made by continues changes on photosphere.

\section{Acknowledgements}

We'd like to acknowledge the BBSO and Huairou Observing Station for the data used. Supported by National Key Basic Research Science Foundation of China (G2000078404) and National Natural Science Foundation of China (10233050). The author appreciate the help from Yuzong Zhang and Hui Zhao.

\section{References}

Wang, J.X., Shi, Z.X., Wang, H.N. \& Lu, Y.P. 1996, ApJ. 456, 861

Deng, Y.Y., Wang, J.X., Yan, Y.H. \& Zhang, J. 2001, Solar Physics 204, 13

Leka, K.D. \& Barnes, G. 2003, ApJ, 595, 1277

Wang, J.X., Zhou, G.P. \& Zhang, J. 2004, ApJ. 615

Hagyard, M.J., Moore, R.L. \& Emslie, A.G. 1984, Solar Physics 4, 71

Zhang, J., Wang, J. et al. 2001, ApJ. 548, 99 